Mikołaj NKOLLO

Université Adam Mickiewicz de Poznań

\title{
LES CONVERSES DE RELATION, LES EXPRESSIONS SYMÉTRIQUES ET LA FLEXION ${ }^{1}$
}

\section{Objet de l'étude}

Dès la naissance de la linguistique moderne, les études flexionnelles attirent invariablement l'intérêt des chercheurs. Cependant, ceux qui prennent position devant les problèmes relatifs à ce domaine privilégient, à peu près unanimement, le côté formel des unités susceptibles d'être fléchies, en même temps qu'ils passent sous silence leurs aspects sémantiques. Une telle attitude est commune aux chercheurs se réclamant de diverses orientations théoriques et méthodologiques. Les linguistes, aussi bien ceux qui considèrent le mot comme le principal objet de l'analyse flexionnelle (CREIDER et HUDSON, 1999 : 166-169) que ceux qui favorisent le paradigme (voir STUMP, 2001 : 33-36), évitent d'étendre leurs recherches aux problèmes sémantiques. Il se crée de la sorte et indépendamment de la langue étudiée une vision appauvrie du fonctionnement des unités flexionnelles. Le but de cet article consiste à montrer que celles-ci possèdent également des caractéristiques sémantiques permanentes. En outre, il s'agit de découvrir ce qui met en correspondance, sur le plan sémantique, des unités formellement non apparentées entre elles. Les observations qui suivent ont un caractère général et concernent toutes les langues où la forme des unités lexicales est susceptible de variations catégorielles.

Le texte commence par des remarques concernant les converses de relation. Après une brève esquisse $\mathrm{du}$ traitement que ces expressions reçoivent généralement dans des ouvrages de linguistiques contemporains, il est question de certaines relations flexionnelles jugées utiles pour la détermination de leur statut paradigmatique. La partie consacrée à l'analyse des expressions symétriques est construite selon un même principe - la définition de cette classe de signes est suivie de la présentation des problèmes que connaît leur analyse formelle. La solution consiste, une nouvelle fois, à évoquer les notions empruntées à la théorie générale de flexion.

\subsection{Les converses de relation - définition et problèmes de description}

L'épanouissement de la sémantique a permis de découvrir que l'information sur un état de choses peut être transmise par des unités et par des constructions morphologiquement et syntagmatiquement éloignées les unes des autres. En témoignent les exemples :

\footnotetext{
${ }^{1}$ Je tiens à remercier les personnes qui ont pris la peine de lire mon article et d'y apporter leurs commentaires. Ils m'ont été fort utiles lors de la rédaction du texte. Je suis l'unique responsable d'éventuelles erreurs et incorrections.
} 


\section{1a. Ce manoir appartenait à ma famille depuis des siècles}

1b. Ma famille possédait ce manoir depuis des siècles

La première de ces phrases asserte exactement la même chose que la seconde il y a deux individus et la relation qui les relie est, dans l'un et dans l'autre cas, la même. Il est donc question ici d'un seul état de choses, ceci en dépit de la différence formelle qui sépare appartenir et posséder.

Les deux phrases justement analysées sont fondées sur la présence des converses de relation. Les sémanticiens qui entreprennent la description de celles-ci se contentent de formuler l'opinion sur la primauté de la sémantique par rapport à la morphologie. Cette dernière ne serait, à leurs yeux, qu'un reflet superficiel de certaines régularités sémantiques jugées universelles. En effet, chaque langue abonde en constructions qui, en dépit des caractéristiques formelles qui les séparent, ne diffèrent pas au niveau de ce qu'elles signifient. Cependant, lorsqu'on met laccent uniquement sur les composantes sémantiques d'un terme et qu'on se limite à l'énumération de ses synonymes, on risque de négliger d'entrevoir les régularités formelles permettant d'exprimer un même sens. Il est donc urgent d'essayer de marier les deux courants de recherche : la description sémantique des phrases d'une langue naturelle et la description paradigmatique des termes responsables de l'existence de la synonymie entre elles.

Les converses de relation sont définies de la façon suivante dans les ouvrages s'inspirant de la logique formelle (c'est à partir de ce domaine que la notion a pénétré dans les recherches linguistiques) :

2. $x R^{-1} y$ si et seulement si $y R x^{2}$

Jugée peu utile par certains chercheurs (APRESJAN, 2000 : 243), cette définition révèle ce qui est essentiel dans la description des converses de relation. Premièrement, la notation $R^{-1}$ et $R$ suggère que l'existence d'au moins deux termes est nécessaire. Cela veut dire qu'une seule expression n'est jamais, à elle seule, une converse de relation. Elle est toujours la converse de la relation désignée par un autre terme. Ce dernier forme, à son tour, une paire de converses de relation avec l'expression de départ. Or, la notion discutée ici a clairement un caractère relationnel.

L'autre propriété linguistique des termes en question, inscrite dans la définition, consiste dans la différence formelle des membres de la paire. Ce trait

\footnotetext{
${ }^{2}$ Pour appliquer cette formule à une langue naturelle, il faut déchiffrer les signes qui la constituent. Les lettres $x$ et $y$ représentent les variables individuelles. Autrement dit, ce sont les symboles capables de désigner un des éléments d'un ensemble. Il faut souligner qu'une variable individuelle ne réfère pas à tous les membres de cet ensemble, mais à un objet non spécifié qui en fait partie (BAŃCZEROWSKI, 1980 : 12). Il existe deux façons d'attribuer à une variable sa dénotation. La première consiste à lui substituer une constante, c.-à-d. à spécifier expressément ce qui est représenté par la variable (p. ex. Max pour $x$ et Jacqueline pour $y$ ). L'autre façon de préciser sa valeur référentielle est due à l'emploi des opérateurs qui lient les variables présentes dans la fonction propositionnelle soumise à la transformation (p. ex. les quantificateurs). Ces deux opérations permettent de transformer la fonction (séquence de symboles contenant les variables) en une proposition au sens logique du terme (suite de signes, douée d'une valeur de vérité, c.-à-d. vraie ou fausse). Par la suite, la transformation réalisée à l'aide d'une de ces opérations sera nommée saturation.
} 
est extrêmement important. Comme on le sait, les langues naturelles abondent en expressions symétriques dont la particularité consiste à admettre l'interversion des éléments nominaux, sans qu'il soit nécessire d'altérer la forme du terme prédicatif (Nous ne sommes pas d'accord avec vous équivaut, du point de vue de ses conditions de vérité, à Vous n'êtes pas d'accord avec nous). Ce type de signes linguistiques sera étudié dans la deuxième partie du présent article. On voit que l'effet structural (le changement des positions des éléments nominaux) imputable à la présence des expressions symétriques est identique à celui que produisent les converses de relation. Cependant, la notation $R^{-1}$ et $R$ suggère qu'il doit y avoir, lorsqu'on a affaire à celles-ci, une différence formelle à l'intérieur de la paire.

Ce statut linguistique des converses de relation pose au chercheur le problème de savoir s'il est possible de les intégrer dans la description flexionnelle d'une langue. L'idée de marier l'analyse sémantique et les recherches paradigmatiques peut paraître étrange. Le champ d'investigation de la flexion, à savoir les modifications des formes d'un seul lexème, n'exhibe apparemment aucun lien systématique avec l'analyse des signes qui s'apparentent du point de vue de leurs sens, mais qui s'éloignent formellement les uns des autres.

\subsection{Les aspects flexionnels du fonctionnement des converses de relation}

Dans la tradition linguistique, l'importance de la flexion se manifeste là où il faut décider à quel moment nous avons affaire à une seule unité lexicale (dont les membres appartiennent à un seul paradigme) et en particutier quand il y a création d'unités nouvelles dont le champ d'investigation est la dérivation. Les chercheurs s'accordent à admettre que les modifications catégorielles ne donnent pas naissance à plusieurs unités lexicales distinctes (cf. toutefois le point de vue de BYBEE, 1985 : 91-95). Ceci explique pourquoi les formes animal et animaux, je et me ne sont pas classifiées individuellement dans les dictionnaires. Il s'agit, dans chacune de ces paires, de variantes flexionnelles émanant d'un lexème. On note, en même temps, que toutes les unités variables sont, dans les langues indoeuropéennes, obligatoirement porteuses d'une des valeurs catégorielles discernables dans la partie du discours qu'elles représentent. L'expression de ces valeurs est assurée justement par la flexion. Celle-ci est donc obligatoire dans toutes les unités suceptibles de variations catégorielles. C'est ainsi que chaque nom polonais transmet obligatoirement l'information sur le cas, le nombre, en même temps qu'il représente un des genres (cf. BAŃKO, 2002 : 142-148). Le désintéressement dont souffrent les converses de relation parmi les linguistes qui s'occupent de la flexion paraît donc justifié.

Toutefois, les objets de la description flexionnelle sont souvent, en ce qui concerne leurs propriétés formelles, extrêmement diversifiés. Des pages entières sont consacrées, dans les ouvrages scolaires, à l'énumération des irrégularités qu'exhibent les pluriels de certains noms ou les formes temporelles, aspectuelles, etc. dans la morphologie verbale. L'originalité des paradigmes de ces mots est qu'ils s'appuient sur plusieurs radicaux. C'est cette pluralité qui est responsable de l'hétérogénéité de leurs formes flexionnelles. En outre, dans plusieurs langues humaines, l'expression de chaque catégorie connaît une diversification analogue. 
C'est ainsi que les éléments nominaux polonais peuvent différer par leurs thèmes en nombre (człowiek « homme » vs. ludzie « gens »), en cas (on nom. sing. vs. go acc. sing.), etc. Il en est de même de la plupart des catégories verbales polonaises et de la gradation des adjectifs et des adverbes. Le terme "hétérothématique »va être employé par la suite pour nommer les unités lexicales dont les paradigmes connaissent plusieurs thèmes. Ces unités s'opposent par les propriétés morphologiques de leurs variantes flexionnelles aux unités homothématiques (qui s'appuient sur un seul radical), qualifiées de « régulières ».

La relation entre les formes flexionnelles appartenant au paradigme d'un lexème hétérothématique est appelée suppléance. Elle est symétrique (s’il y a suppléance entre mieux et bien, elle se manifeste aussi entre bien et mieux). L'ensemble des formes flexionnelles appartenant au paradigme d'un lexème hétérothématique constitue le domaine de variabilité de cette relation (BAŃCZEROWSKI, 1999: 489). Les recherches typologiques et comparatives confirment que la suppléance affecte surtout les paradigmes des unités enracinées depuis longtemps dans une langue (FLEISCHMANN, 1982 : 35-37, JOSEPH, 1998 : 367-368). Les formations néologiques et les emprunts représentent généralement les modèles flexionnels pleinement réguliers. Il n'est alors pas surprenant de découvrir que être et avoir sont considérés comme les moins réguliers dans la morphologie verbale française ou qu'il existe une différence de radicaux dans la gradation de bon (bien) et partiellement dans celle de mauvais (mal). Enfin, l'opposition formelle que l'on observe entre le singulier homme et le pluriel gens confirme le caractère particulier de l'évolution des unités lexicales le plus fréquemment employées, donc les moins spécialisées (LAMIROY, 1999 : 37).

Le fait que le paradigme d'une unité lexicale puisse reposer sur plusieurs thèmes permet de supposer qu'il existe, au niveau flexionnel, certaines relations systématiques entre les membres d'une paire de converses de relation. La validité de cette opinion est confirmée par l'observation des propriétés sémantiques qui doivent caractériser deux variantes morphologiques d'un même lexème. La première d'entre ces propriétés, qui va être appelée homosignification, renvoie à la présence d'un invariant sémantique, une partie de sens commune à toutes les formes flexionnelles d'une unité lexicale. Lorsqu'on décline un nom, il est facile de repérer ce qui, en dépit de la différence des terminaisons ou des radicaux, rapproche toutes ses formes flexionnelles - il est question en ce lieu de la propriété de signifier un même objet. Cette même constance s'observe également dans la morphologie verbale, ceci en dépit de la diversification des formes représentant diverses valeurs catégorielles des unités telles que être ou avoir. Le critère d'homosignification est donc nécessaire à l'intégration de plusieurs formes dans un paradigme.

Toutefois, l'homosignification, à elle seule, ne suffit pas à dégager deux variantes d'une unité lexicale. Deux formes sémantiquement apparentées (p. ex. obstination et entêtement) sont encore loin de pouvoir former un paradigme ; il n'y a pas de corrélation flexionnelle entre elles. Pour qu'une telle corrélation ait lieu, il faut que les différentes formes d'un seul lexème s'opposent sémantiquement. Le simple bon sens dit d'ailleurs qu'il n'y a pas grand intérêt à les diversifier là où elles continuent à assumer les mêmes fonctions sémantiques et signifient 
exactement la même chose. L'invariabilité est contradictoire à ce qui consitue l'essence même d'un paradigme ( « In the most general sense flectional relations are of partial similarity, that is, they bind phrases which simultaneously exhibit both : (i) equality with respect to certain properties, and (ii) distinction with respect to others [...] Equality ensures an indispensable foundation for distinctions to operate as flectional indicators. And, hence, flection appears as a subclass of opposition », cf. BAŃCZEROWSKI, op. cit.: 484). Le même principe est valable pour tous les signes linguistiques susceptibles d'être fléchis. Les mots latins pirată, piratae, piratam ou piratā, bien qu'ils transmettent invariablement l'information sur un même objet, s'emploient dans différents contextes syntagmatiques et leurs rôles sémantiques diffèrent les uns des autres. Il en est de même des formes supplétives françaises fus et étais ou polonaises będę brat («prendre», fut. imperf.) et wezme (" prendre » fut. perf.). Dans ce dernier exemple, l'une et l'autre forme signifient l'éloignement prospectif d'une seule activité dans le temps, encore qu'elles la représentent différemment.

Puisque deux formes faisant partie d'un paradigme, même celles qui sont supplétives, doivent être sémantiquement apparentées, la question se pose de savoir quelles sont les propriétés partagées par les membres d'une paire de converses de relation. Pour résoudre cette énigme il faut retourner à la définition de cette classe de signes : indépendamment du terme qu'on emploie, les constructions fondées sur la présence des converses de relation signifient un même fragment de la réalité. En outre, si les deux phrases sont falsifiables, leur valeur logique demeure intacte. L'identité des propriétés vériconditionnelles permet de reconnaître si l'on a, oui ou non, affaire à une paire de converses de relation. La construction Jacques a reçu une vive réprimande de Pierre ne peut pas être fausse si Pierre a vivement réprimandé Jacques est vraie. Il est, bien entendu, possible d'échanger l'ordre des phrases de cette formule ; il n'en reste pas moins que les valeurs logiques de l'une et de l'autre doivent être identiques.

Il est plus difficile de déterminer la nature de la diversification sémantique produite par l'emploi des converses de relation. Pourtant, les oppositions de sens sont nécessaires à la délimitation des différentes formes d'un paradigme. Les phrases Le départ de Max a directement précédé cet accident et Cet accident a directement succédé au départ de Max transmettent la même information, mais elles le font chacune à sa manière. La première renvoie à ce qui est arrivé après le départ de Max, l'autre - aux circonstances survenues avant un certain accident. Les deux phrases répondent à des questions différentes (p. ex. Que s'est-il passé après le départ de Max ? et Que s'est-il passé avant cet accident ?). C'est donc surtout au niveau de la structure thème-rhème que s'opposent les constructions fondées sur chacun des membres de la paire. Cependant, ces oppositions ne doivent pas être confinées aux facteurs pragmatiques et discursifs (voir ŻUKROWSKA, 1998 : 71). La modification des propriétés référentielles des membres de la paire est bien visible là où les éléments nominaux qui saturent les positions syntaxiques ouvertes par le terme constitutif sont au pluriel, sont des noms collectifs ou qu'on les soumet à une quantification particulière (ANSCOMBRE, $1996: 85-86$, KEENAN, $2002: 629-633$, WINTER, $2002:$ 495-496). Le changement du statut vériconditionnel des constructions flexionnellement et lexicalement apparentées, consécutif à la pluralisation, a déjà été noté lorsque la 
grammaire générative était en plein essor. Il est question ici des mutations sémantiques imputables à la passivation. La querelle autour des différences qui séparent la construction active et son pendant passif a été provoquée par les fameux exemples de CHOMSKY (1971 : 199-201) - Many arrows hit the target et The target was hit by many arrows. Toutes ces particularités liées au fonctionnement des constructions fondées sur la présence des converses de relation concourent à assurer à celles-ci l'appartenance au paradigme d'un seul lexème hétérothématique.

\subsection{Le statut paradigmatique des converses de relation}

La relation de suppléance, qui concerne l'ensemble des variantes d'un lexème, ne peut pas être évoquée sans quelques indications préalables. L'examen des propriétés morphologiques des objets faisant partie d'un paradigme flexionnel montre clairement qu'ils ne sont pas supplétifs tout court. Il faut, chaque fois qu'on emploie ce qualificatif, préciser la catégorie grammaticale à l'intérieur de laquelle deux formes s'opposent par leurs thèmes. C'est ainsi qu'il y a suppléance dans le paradigme du nom polonais czlowiek « homme » uniquement en ce qui concerne la catégorie du nombre. En revanche, d'autres catégories discernables dans la morphologie nominale polonaise sont exprimées d'une façon régulière, c.à-d. à la base d'un seul thème. En témoignent les paires człowiek-a (gen. sing.) et człowiek-owi (dat. sing.) ou bien ludz-i (gen. pl.) et ludz-iom (dat. pl.). La suppléance casuelle n'existe pas ici. De même, la diversification formelle que l'on observe entre melius et bonus est liée uniquement à l'expression du degré (et non pas du cas ou du nombre, deux autres catégories de l'adjectif latin). Il en résulte que la relation de suppléance, même dans les langues flexionnelles (où les morphèmes catégoriels ont un caractère cumulatif, cf. STUMP, 1998 : 31-32), ne doit pas être appliquée d'une façon absolue. La question se pose alors de savoir quelle catégorie est appropriée à la description du statut flexionnel des converses de relation. La découverte de cette catégorie exige qu'on tienne compte de l'appartenance des converses de relation aux différentes classes morphologiques. Puisque les catégories flexionnelles ne se répètent pas d'une partie du discours à l'autre, il faut que soient examinés les verbes, les noms et les adjectifs.

En ce qui concerne la première classe, les converses de relation entretiennent des liens privilégiés avec la catégorie de la voix dans la morphologie verbale. La propriété syntagmatique essentielle des phrases fondées sur la présence de ces expressions - l'interchangeabilité des positions des éléments nominaux - est identique à celle qu'on observe lors du passage de la construction active à son correspondant passif. Cette caractéristique concerne en principe les phrases comportant un verbe bivalent, accompagné de tous les éléments nominaux requis par ses propriétés combinatoires et sémantiques. L'effet sémantique consécutif à la passivation ou à l'emploi d'un des membres d'une paire de converses de relation au lieu de son équivalent est, lui aussi, identique. On posera donc qu'il existe un parallélisme étroit entre la modification de la hiérarchie des éléments nominaux que produisent le changement de la voix du verbe et l'emploi des converses de relation. 
3a. Le tabagisme provoque les maladies du système de circulation sanguine

3b. Les maladies du système de circulation sanguine sont provoquées par le tabagisme

3c. Les maladies du système de circulation sanguine découlent du tabagisme

Cependant, il faut éviter de réduire les possibilités qu'offre l'emploi des converses de relation au rang d'alternative aux mutations dans la morphologie verbale. L'abandon des confins qu'impose le partage en parties du discours est nécessaire, car les converses de relation ne servent pas uniquement à concurrencer la mutation flexionnelle du verbe. L'effet sémantique mentionné dans la définition a un caractère général et peut être réalisé aussi par les adjectifs et les noms. Comme on le sait, les expressions qui représentent ces deux parties du discours ne connaissent pas, dans les langues romanes, la catégorie de la voix. L'emploi des converses de relation en guise d'alternative à la passivation du verbe n'est donc qu'un cas de figure parmi la multitude des effets structuraux possibles à obtenir également là où la phrase comporte les adjectifs et les noms. Le problème qui se pose est de savoir comment ces deux classes fondent les phrases qui, en dépit de leurs caractéristiques morphologiques divergentes, signifient exactement un même état de choses. Dans un premier temps, l'analyse va se concentrer sur les adjectifs.

\section{4a. Les températures des mois d'été sont plus hautes que celles du mois de septembre}

4b. Les températures du mois de septembre sont moins hautes que celles d'été

4c. Les températures du mois de septembre sont plus basses que celles du mois d'août

Du point de vue morphologique, c'est le degré comparatif qui est en cause ici. L'exposant flexionnel de cette valeur catégorielle plus (moins)... que assume la fonction de variable prédicative et ouvre une position syntaxique au nouvel élément nominal. Les adjectifs haut et bas, employés dans les phrases ci-dessus, ne se limitent pas à nommer la propriété attribuée à certains objets extralinguistiques (comme ceci a lieu là où l'on a affaire au degré positif, cf. La Tour Eiffel est haute, mais non La Tour Eiffel est haute que cet immeuble). Le rôle de plus (moins)... que consiste à introduire l'idée de comparaison dans le sens véhiculé par l'adjectif (KAROLAK, 1965 : 148-149). La comparaison étant une relation symétrique, elle est opératoire dans les ensembles qui comportent au moins deux éléments (KRĄPIEC, 1985 : 230 et ff.). Les propriétés syntagmatiques des constructions comparatives sont donc particulièrement favorables à l'emploi des converses de relation qui renvoient, elles aussi, aux situations auxquelles participent au moins deux individus. Il doit être souligné que, dans les exemples 4a-c, ce n'est pas exclusivement l'adjectif qui est membre d'une paire de converses de relation. Il assume cette fonction uniquement à condition d'être accompagné des morphèmes du comparatif.

L'autre propriété des adjectifs dont il faut tenir compte ici est liée à leur statut syntaxique. Uniquement là où ils sont attributs, les adjectifs assument la fonction du prédicat et, ce qui en résulte, fondent les constructions qui, en dépit des différences structurales et lexicales qui les séparent, signifient invariablement un 
état de choses. Il y a donc, dans la flexion adjectivale, des liens privilégiés entre la catégorie du degré et l'emploi des termes homosignificatifs et, en même temps, hétérothématiques.

Les converses de relation peuvent enfin revêtir la forme nominale. Il est, en premier lieu, question ici des paires de noms abstraits qui requièrent la saturation de deux positions syntaxiques. Ces expressions permettent de construire les syntagmes complexes où le nom abstrait assume la fonction de déterminé absolu. Les constructions ainsi formées peuvent assumer différentes fonctions syntaxiques dans la structure de la phrase. En ce qui concerne le statut référentiel de ce groupe de noms, ils sont clairement syncatégorématiques. Cela veut dire qu'ils sont incapables de donner naissance, eux mêmes, à des actes de désignation autonomes. Ce n'est qu'à condition de leur adjoindre leurs compléments nominaux qu'ils acquièrent la possibilité de dénommer, de renvoyer à des objets identifiables de la réalité extra-linguistique. Dans les langues à articles, l'acquisition par ces noms d'un caractère catégorématique est signalée par la présence du prédéterminant défini (cf. une victoire et la victoire des troupes fidèles au gouvernement sur la guérilla maö̈ste vs. une défaite et la défaite infligée à la guérilla maoïste par les troupes fidèles au gouvernement).

Les noms de relation constituent une autre classe d'expressions homosignificatives capables de former des paires de converses de relation. Ils s'opposent au groupe précédent aussi bien par leurs propriétés sémantiques que par leurs propriétés combinatoires. Les entités qu'ils désignent ne sont pas abstraites ; bien au contraire, ce sont des objets individuels. En ce qui concerne le niveau syntagmatique, les noms de relation postulent la présence d'un seul complément (cf. GIRY-SCHNEIDER, 1997 : 24). Enfin, ils ne forment des paires de converses de relation qu'à condition d'assumer la fonction d'attribut (Pierre est le mari d'Irène et Irène est la femme de Pierre ou Stendhal est l'auteur de "Le rouge et le noir " et "Le rouge et le noir " est l'œuvre de Stendhal). L'analogie qu'ils exhibent avec les noms abstraits consiste dans leur caractère syncatégorématique.

L'examen de différentes parties du discours confirme les difficultés que pose l'identification de la catégorie flexionnelle appropriée à la description des converses de relation en termes de suppléance à l'intérieur du paradigme d'un seul lexème. L'explication de l'énigme consiste à découvrir ce que les verbes, les adjectifs et les noms, indépendamment des particularités morphologiques qu'ils présentent, ont en commun. Le mérite des linguistes qui utilisent la notion de converse de relation dans leurs travaux consiste à l'avoir localisée dans le domaine des recherches sur les modèles syntagmatiques imposés à leur entourage par les termes prédicatifs. Comme on le sait, les langues naturelles disposent de tout un éventail de moyens morphologiques et syntaxiques permettant, sans faire varier les unités lexicales employées, de renvoyer à un même état de choses. Cette multiplicité est obtenue grâce aux diverses configurations des termes qui saturent les positions syntaxiques ouvertes par l'expression prédicative. L'ensemble de ces configurations est appelé diathès e.

Bien entendu, l'équivalence sémantique des constructions comptées au nombre des modèles diathétiques d'une langue n'est pas parfaite. C'est ainsi qu'à côté de la voix passive, privilégiée par la tradition grammaticale et construite à l'aide de la 
séquence être suivi du participe passé (p. ex. être vendu), le français recourt parfois, pour rendre compte d'un même état de choses, à la forme finie du verbe accompagnée d'un pronom (se vendre). On le voit bien dans les exemples cidessous.

5a. Les fermiers vendent le blé à perte

5b. Le blé est vendu à perte par les fermiers

5c. Le blé se vend à perte

Dans la dernière de ces phrases, il est obligatoire de supprimer un élément nominal, la séquence Le blé se vend à perte par les fermiers étant incorrecte. En même temps, les chercheurs sont d'accord pour ne pas reconnaître à cette phrase un sens réfléchi (comme dans Elle se vend au premier venu) ou réciproque (Ils se vendent les voitures l'un à l'autre). C'est pour cette raison que les constructions du type Le blé se vend à perte sont souvent considérées comme sémantiquement équivalentes à la voix passive (KEENAN, 1985 : 257-259). Il en est de même de l'emploi de se faire (Les conquérants massacrent les autochtones, Les autochtones sont massacrés par les conquérants, Les autochtones se font massacrer par les conquérants), de voir (Paul reviendra dans l'après-midi et L'après-midi verra Paul revenir ; cf. BAT-ZEEV SHYLDKROT, 1987 : 230 et ff.) et de plusieurs autres verbes.

Cependant, la richesse des modèles syntagmatiques permettant d'exprimer un même sens n'en finit pas là. Lorsqu'on dresse la liste de ces modèles, il est nécessaire qu'on tienne compte de l'existence des converses de relation hétérothématiques, car l'effet sémantique que ces expressions produisent est identique à celui qu'on observe lors du passage d'une forme flexionnelle (voix active) à l'autre (voix passive). Il est donc légitime de parler de l'existence d'un paradigme diathétique. En outre, le fait que, dans le discours quotidien, les énoncés contiennent le plus souvent une forme finie du verbe, n'implique pas que les variantes diathétiques doivent être nécessairement, toutes, de nature phrastique. L'occurrence d'un nom abstrait en compagnie de ses arguments donne lieu, elle aussi, à l'étude des modèles syntagmatiques imposés par une expression prédicative à son entourage. Il en est ainsi car la notion de diathèse renvoie aux diverses configurations des termes qui saturent les positions syntaxiques ouvertes par le terme central, indépendamment de la nature morphologique de celui-ci. On ne s'étonne donc pas de la capacité des noms abstraits à constituer le déterminé absolu de ces constructions (cf. l'influence de ses professeurs sur son psychisme, l'influence que subit son psychisme de la part de ses professurs, l'influence qu'exercent ses professeurs sur son psychisme, et d'autres). À l'opposé de ce qui est généralement pratiqué dans la littérature linguistique, cet article défend l'opinion que la notion de diathèse ne doit pas être appliquée exclusivement à la forme que revêtent les phrases entières. Même si une construction est dépourvue de la forme finie du verbe et même si son articulation ne s'accompagne pas d'un contour intonatif approprié, il est légitime de parler de diathèse. L'unique condition est qu'on puisse délimiter, à l'intérieur de la construction soumise à l'analyse, un élément prédicatif et les arguments susceptibles de saturer les 
positions syntaxiques qu'il ouvre (ou, au moins, une partie d'entre eux, cf. LEFEUVRE, 1999 : 15).

Cette solution permet d'attribuer aux paires de noms abstraits et de noms de relation leur place parmi la multitude des variantes syntagmatiques réalisant un même sens. Les converses de relation peuvent donc être légitimement considérées comme les formes supplétives à l'intérieur du paradigme diathétique qu'une langue met en place pour signifier un même état de choses (« ... the flectional domain is not properly included in that of morphology, but both domain intersect, and dictonal flection should be located within this intersection. Sentential flection will in fact belong to the category of diathesis », BAŃCZEROWSKI, op. cit. : 484). C'est pour cette raison que les paires de converses de relation ne sont pas formées uniquement par les expressions appartenant à une même partie du discours (p. ex. auteur et ouvrage ou précéder et succéder à / suivre). La suppléance a lieu également là où l'on apparie les expressions nominales, adjectivales ou verbales, les unes avec les autres. Le verbe précéder n'est donc pas la converse de la relation signifiée uniquement par suivre ou succéder à. Il est supplétif aussi par rapport à postériorité, postérieur, succession, etc. Les facteurs sémantiques sont décisifs de ce point de vue (KAROLAK, 2002 : 63).

\subsection{Les relations symétriques - définition et problèmes de description}

En ce qui concerne la place des expressions symétriques dans la description flexionnelle d'une langue, l'analyse commencera une nouvelle fois par la définition de cette classe. La symétrie renvoie à la propriété qu'ont certains termes de désigner les relations qui, si elles sont vraies pour $x$ et $y$ pris dans cet ordre, doivent l'être également pour $y$ et $x$. Ceci veut dire que la vérité de la relation (représentable à l'aide du schèma) « $x R y$ » implique également la vérité de « $y R x »$, pour tous les $x$ et tous les $y$ faisant partie d'un même ensemble. D'une façon formelle, la symétrie se présente donc comme dans 6 .

$$
\text { 6. } \operatorname{sym}(X)={ }_{\mathrm{df}}\{R: \forall x \forall y(x, y \in X \wedge x R y \rightarrow y R x)\}
$$

Comme précédemment, cette définition est une fonction propositionnelle contenant des variables. $R$, symbole prédicatif, doit être saturé par une expression (nom, adjectif, verbe, collocation verbo-nominale du type faire cul et chemise avec ou avoir des atomes crochus, etc.) précisant la nature du rapport entre $x$ et $y$ (ou entre les référents des noms qui ont été introduits à leur place). L'effet structural qu'entraîne la présence d'une expression symétrique, c.-à-d. le changement des positions syntaxiques des éléments nominaux, est identique à celui qu'on doit à l'emploi des converses de relation. La première propriété syntagmatique des expressions en question est inscrite dans la définition. La relation dont elle parle est nécessairement dyadique. Autrement dit, pour qu'elle ait lieu, la présence de deux participants est requise. Il s'ensuit que les prédicats qui ouvrent seulement une position d'argument (dormir, éternuer, etc.) ne sont jamais symétriques. En outre, l'emploi d'une expression symétrique exige que chacun des arguments soit mentionné. L'omission de l'un d'entre eux entraîne la mauvaise formation grammaticale de l'énoncé, cf. *l'analogie entre ce livre (au lieu de p. ex. 
l'analogie entre ce livre et le récit de son père). L'omission du nom de l'autre participant à la relation abolit l'autonomie référentielle des constructions comme *l'analogie entre ce livre.

La caractéristique sémantique essentielle des expressions symétriques consiste à établir la parité de ce qui est prédiqué de l'un et de l'autre participant à la relation. Celle-ci, une fois vraie pour l'ensemble $\langle x, y\rangle$, il doit en être de même là où les termes en question concernent les membres respectifs de $<y, x>^{3}$. En termes de sémantique lexicale, cela signifie que les prédicats symétriques instituent l'égalité entre les référents de leurs arguments et renseignent sur l'homogénéité de leurs actions. Un seul état de choses est donc désigné aussi bien par Son point de vue est différent de celui de sa femme et Le point de vue de sa femme est différent du sien (BECK, 2000 : 102-106).

La question surgit donc de savoir s'il est possible de donner une description flexionnelle aux expressions formellement identiques et homosignificatives qui ont la propriété de fonder les phrases qui ne diffèrent entre elles que par l'ordre de leurs éléments nominaux. Bien que les valeurs logiques de Pierre est de la même taille que Max et de Max est de la même taille que Pierre soient identiques, certaines différences sémantiques et pragmatiques s'observent entre elles. C'est, une nouvelle fois, au niveau de la structure thème-rhème qu'elles peuvent s'opposer. En outre, les statuts vériconditionnels de ces deux constructions peuvent être différents là où elles sont subordonnées à une principale contenant certaines indications modales. En témoignent les exemples La grand-mère a demandé si Pierre était de la même taille que Max et La grand-mère a demandé si Max était de la même taille que Pierre. Dans une des interprétations possibles, le premier exemple renvoie à la situation où c'est Pierre qui est au centre d'intérêt de la grand-mère. Dans le second cas, la situation est inverse.

Les phrases fondées sur la présence des expressions symétriques, bien qu'elles exhibent certaines différences réelles au niveau sémantique, restent homosignificatives. L'une et l'autre caractéristique sont nécessaires pour regrouper ces expressions dans un paradigme. Il est possible d'objecter à ce raisonnement qu'il surestime les conséquences d'un effet syntagmatique banal et qu'il postule, à tout prix, l'existence de deux lexèmes là où, en réalité, il n'y en a qu'un seul. Cet effet syntagmatique est imputable à la propriété qu'ont les expressions symétriques de prédiquer exactement la même chose de l'un et de l'autre participant à la relation (NKOLLO, 2003 : 108-109). Cependant, les différences sémantiques, soient-elles infimes, entre les phrases où l'ordre des éléments nominaux est permuté montrent assez clairement qu'une telle critique n'est pas bien justifiée. Mais, cette preuve n'est pas la seule. Une autre, plus importante, est due à l'analogie entre l'analyse flexionnelle des converses de relation et celle des expressions symétriques. Si les premières possèdent leur place

\footnotetext{
${ }^{3}$ La formule $\langle x, y>$ ne doit pas être confondue avec la notation d'un ensemble ordinaire, réalisée à l'aide des accolades $\{x, y\}$ parce que leurs statuts ontologiques diffèrent sensiblement. Tandis que l'ensemble $\{x, y\}$ équivaut à $\{y, x\}$ ou à $\{x, x, y, y, x\}$, il n'en est pas de même de l'entité représentée $\operatorname{par}\langle x, y\rangle$. Celle-ci est un ensemble ordonné d'éléments qui ne sont pas permutables. Il n'est pas non plus possible de les répéter. Le recours aux ensembles ordonnés de $n$ éléments est nécessaire si l'on veut définir ce que c'est qu'une relation. Cette notion doit être décrite en termes des ensembles des paires ordonnées d'éléments (cf. CANN, 1993 : 50).
} 
dans la description morphologique, pourquoi ne doit-il pas en être de même avec les secondes ? Ni l'une ni l'autre classe d'expressions ne constituent un microsystème lexical hermétique. Bien au contraire, leur statut est comparable à celui des autres expressions. C'est pourquoi les converses de relation et les expressions symétriques méritent d'obtenir la même analyse que d'autres signes faisant partie du système lexical d'une langue.

\subsection{Les relations flexionnelles utiles dans l'analyse des expressions symétriques}

La correspondance morphologique entre les membres d'une paire de converses de relation, qui s'éloignent les uns des autres par leurs formes, s'est établie grâce à la notion de suppléance à l'intérieur du paradigme diathétique. Quel est donc le statut flexionnel des expressions symétriques, qui ne présentent pas d'oppositions formelles ? Les recherches sur la flexion de plusieurs langues du monde ont abouti à la découverte de la coexistence de deux (ou parfois plusieurs) formes identiques à l'intérieur d'un seul paradigme. Cette identité est appelée, dans la tradition linguistique, syncrétisme. Il convient donc de vérifier si cette notion peut être appliquée à la description du statut flexionnel des expressions symétriques.

Le syncrétisme est une homonymie complète entre au moins deux formes flexionnelles d'une unité lexicale. Il a donc toujours lieu à l'intérieur du paradigme d'un terme variable (ARONOFF, $1994: 83-84)^{4}$. Cette définition montre clairement que le syncrétisme est une relation symétrique; s'il a lieu entre une forme grammaticale $x$ et une autre forme $y$, il doit nécessairement être valable pour l'ensemble $y$ et $x$, pris dans cet ordre. En français, le fonctionnement de cette régularité peut être démontré par l'exemple de la $1^{\text {ère }}$ et de la $3^{\text {ème }}$ personne du singulier (indicatif et subjonctif présent) des verbes formés par l'adjonction de -er à la racine (je / il regarde, je / il lève, etc.). Les syncrétismes embrassant une seule forme flexionnelle n'existent pas. Il y a également des situations où l'homonymie concerne plus que deux formes flexionnelles à l'intérieur d'un paradigme. Cet état de choses s'observe, d'ailleurs non exceptionnellement, dans plusieurs langues. C'est ainsi que dans la conjugaison anglaise les formes verbales de toutes les personnes sont identiques au pluriel (we / you / they see / saw / have seen, etc.). Il en découle que le syncrétisme (scrt) est également une relation transitive.

Ce raisonnement permet de préciser les différences entre l'homonymie et le syncrétisme. L'homonymie étant une relation d'équivalence (réflexive, symétrique

\footnotetext{
${ }^{4}$ Cette remarque, bien qu'elle puisse sembler banale, doit nécessairement faire partie de la définition du syncrétisme. Certains chercheurs reconnaissent sa présence non seulement à l'intérieur d'un paradigme. Ils confèrent la propriété d'instituer la relation de syncrétisme également aux formants grammaticaux récurrents dans plusieurs catégories grammaticales. Ce problème est discuté par LeISS (1997 : 134-138) à l'exemple du formant anglais $-s$. Celui-ci fonctionne en guise d'exposant du pluriel dans la morphologie nominale. En outre, il est capable de conférer au nom qu'il modifie la valeur de possesseur. Enfin, il sert à former la troisième personne du singulier de certains tiroirs verbaux. Leiss attribue à $-s$ une valeur sémantique sous-jacente, commune à tous ses emplois. Ce point de vue est lié au principe «one form, one meaning » (un des dogmes de la Morphologie Naturelle, cadre méthodologique dont l'auteur se réclame). La valeur sémantique en question est «+Relation ». Ceci s'explique par le fait que le possessif et le pluriel mettent en relation les parties et les totalités, tandis que la $3^{\text {eme }}$ personne du singulier exprime des relations anaphoriques. Ce point de vue, certainement très audacieux, ne sera pourtant pas retenu ici (voir aussi KILBURY, $2001: 341$ ).
} 
et transitive), elle est inclusive par rapport au syncrétisme. Autrement dit, chaque syncrétisme est une instance d'homonymie, mais il y a des homonymies qui ne concernent pas les paradigmes flexionnels. Exemples : voile (nom) et (il) voile (de voiler), grève (nom masculin) et grève (féminin) sont des homonymes. Par contre, civibus (datif pluriel) et civibus (ablatif pluriel) ou copine ( $1^{\text {ère }}$ personne $\mathrm{du}$ singulier) et copine ( $3{ }^{\text {ème }}$ personne du singulier) sont des instances d'homonymie et, en même temps, des syncrétismes flexionnels. Il s'agit des variantes paradigmatiques de civus et de copiner.

Enfin, le dernier problème qu'il est nécessaire d'éclairer lorsqu'on parle de syncrétisme est lié à la complétude de l'homonymie entre deux formes flexionnelles. Celle-ci concerne non seulement la forme phonétique de deux unités. Elle s'étend également à l'ensemble des traits linguistiques pertinents pour l'identification du sens d'un terme dans la chaîne parlée. Cette précision acquiert son importance dès qu'on se souvient des langues où l'accent peut se déplacer d'une syllabe à l'autre ou bien de celles où c'est la quantité vocalique qui a le caractère distinctif. C'est ici que se situe la différence qui sépare les formes latines piratā (ablatif) et pirată (nominatif) et les termes russes мука et мука (suivant que l'accent est oxytonique ou paroxytonique, ces mots signifient " farine » et « tourment », respectivement).

Il convient de réfléchir ici sur la façon dont il est possible d'exploiter la notion de syncrétisme pour déterminer le statut linguistique des expressions symétriques. Pour y aboutir, il faut que soient examinés les modèles syntagmatiques des constructions comportant ces expressions. Leurs propriétés diffèrent, une nouvelle fois, suivant qu'on a affaire à un verbe, un adjectif ou à un nom. En ce qui concerne les verbes symétriques, il est possible d'inverser l'ordre des arguments qui saturent les positions ouvertes par ce groupe de termes (Le départ de Marc coïncide avec mon anniversaire et Mon anniversaire coüncide avec le départ de Marc). Le changement syntagmatique ainsi réalisé ne modifie pourtant nullement le sens des phrases. Leur valeur de vérité reste intacte. Cet effet ne diffère donc pas de celui qu'on attribue à la passivation des phrases à la voix active - s'il est vrai que Les ouvriers ont construit l'école, il doit être nécessairement vrai que L'école a été construite par les ouvriers.

Il faut cependant se garder de conclure trop hâtivement à la parenté des changements produits par l'emploi des expressions symétriques et de ceux qui sont imputables à la modification de la voix verbale. De même que dans des phrases fondées sur la présence de converses de relation, les prédicats symétriques sont actualisés par les expressions appartenant à plusieurs parties du discours. Nous avons vu qu'il existe des liens étroits entre les converses de relation et l'emploi du degré comparatif dans la morphologie adjectivale. Qu'en est-il des adjectifs symétriques ? Les exemples 7 et 8 semblent particulièrement éclairants de ce point de vue.

7a. Notre situation est comparable à la vôtre

7b. Votre situation est comparable à la nôtre

8a. *Jacques est plus égal à sa mère

8b. *Jacques est moins égal à son père 
Comme il a déjà été dit, l'emploi des expressions prédicatives symétriques établit l'égalité de ce qu'on prédique de chaque participant à la relation. Dès lors qu'on asserte à propos d'une chose qu'elle est égale à une autre, on attribue, du même coup, à cette dernière la même caractéristique. Cependant, le recours à un comparatif a pour effet de supprimer l'égalité des objets mis en relation. L'un d'entre eux se voit attribuer plus (ou moins) de propriétés que l'autre. La réalité extra-linguistique ne connaît, bien sûr, pas d'égalité à des degrés non égaux.

Enfin, les relations symétriques sont souvent exprimées par des noms, cf.

9a. La fusion de deux sociétés commerciales

9b. La fusion de la société $X$ avec la société $Y$

9c. La fusion de la société $Y$ avec la société $X$

9d. La fusion de la société $X$ et de la société $Y$

9e. La fusion de la société Y et de la société $X$

9f. Leur fusion

10. Une dysharmonie frappante entre les deux parties de l'ouvrage

La découverte de la catégorie à l'intérieur de laquelle les expressions symétriques seraient des instances de syncrétisme flexionnel consiste, de nouveau, à évoquer la notion de diathèse. Puisque l'emploi de ces termes permet de modifier la disposition des éléments nominaux d'une phrase ou d'un syntagme sans influencer le sens de celle-ci, on a affaire à un changement diathétique. En outre, le statut flexionnel des expressions symétriques se constate grâce aux différences sémantiques entre les constructions fondées sur la présence de ce groupe de termes. D'un autre côté, la comparaison des phrases Jean et Paul sont copains et Paul et Jean sont copains ou The men with the women won one hundred USD et The women with the men won one hundred USD prouvent la quasi-synonymie consécutive à l'emploi de cette classe de signes linguistiques (DALRYMPLE et als., 1998 : 597-599). L'identité formelle des expressions symétriques dans ces constructions n'est pas étonnante : quoi de plus naturel que de se servir d'une seule forme pour désigner un même état de choses ?

\section{En guise de conclusion}

Plusieurs chercheurs ont conclu à l'existence de mécanismes linguistiques et psychologiques responsables de l'identité des formes appartenant à un seul paradigme flexionnel. Selon certains d'entre eux, la synonymie est une condition nécessaire à la fusion de plusieurs formes, naguère distinctes (cf. SERBAT, 1989 : 279-280). Cette constatation semble d'autant plus convaincante qu'elle a été confirmée par l'étude de nombreuses catégories flexionnelles et que plusieurs langues naturelles ont été examinées de ce point de vue. Bien entendu, la proximité sémantique des deux valeurs discernables à l'intérieur d'une seule catégorie ne garantit pas l'identité des formes qui actualisent ces valeurs (cf. SEILER, 1967 : 74-75). De même, le syncrétisme s'observe parfois là où l'existence des traits sémantiques communs est fort douteuse (cf. la note 4 et le cas discuté par Leiss). 
L'originalité des converses de relation et des expressions symétriques doit être incontestablement cherchée au niveau sémantique : c'est justement leur signification qui permet de les opposer à d'autres types de signes dont dispose le système lexical d'une langue. La description des caractéristiques formelles de ces deux classes d'expressions a donc été mise à l'arrière-plan. Cependant, une telle pratique risque de mettre en place une vision appauvrie de la réalité linguistique. Le langage humain n'est pas un objet de nature exclusivement idéale. Il possède également sa face matérielle. Il serait donc naïf de supposer que les converses de relation et les prédicats symétriques forment un microsystème autonome et hermétique à l'intérieur d'un ensemble plus large d'expressions. Il en est tout autrement - leur statut formel permet, lui aussi, de les rapprocher des autres types de signes linguistiques.

\section{BIBLIOGRAPHIE}

Anscombre, Jean-Claude (1996), Partitif et localisation temporelle, Langue Française 109, Paris, Larousse, pp. 80-103.

APReSJAN, Jouri (2000), Semantyka leksykalna. Synonimiczne środki języka, WrocławWarszawa-Kraków, Zakład Narodowy im. Ossolińskich.

ARONOFF, Mark (1994), Morphology by Itself: Stems and Inflectional Classes, Cambridge, Massachussetts, The MIT Press (Linguistic Inquiry Monograph 22).

BAŃCZEROWSKI, Jerzy (1980), Systems of Semantics and Syntax. A determinational theory of language, Warszawa-Poznań, PWN.

BAŃCZEROWSKI, Jerzy (1999), Diversity of Flection (in:) Maria KLAŃSKA, Peter WIESINGER, Vielfalt der Sprachen. Festschrift für Aleksander Szulc zum 75. Geburtstag, Wien, Edition Praesens, pp. 483-504.

BAŃKO, Mirosław (2002), Wykłady z polskiej fleksji, Warszawa, Wydawnictwo Naukowe PWN.

BAT-ZeEv ShyldKRot, Hava (1987), Les compléments de temps et de lieu sont-ils toujours des compléments circonstanciels, Folia Linguistica. Acta Societatis Linguisticae Europaeae XXI (2-4), Berlin, Mouton de Gruyter, pp. 229-247.

BECK, Sigrid (2000), The Semantics of "Different": Comparison Operator and Relational Adjective, Linguistics and Philosophy 23 (2), Dodrecht, Kluwer Academic Publishers, pp. 101-139.

BYBEE, Joan L. (1985), Morphology. A study of the relation between meaning and form, Amsterdam - Philadelphia, John Benjamins Publishing Company (Typological Studies in Language 9).

CAnN, Ronnie (1993), Formal Semantics. An Introduction, Cambridge, Cambridge University Press.

CHOMSKY, Noam (1971), Deep Structure, Surface Structure and Semantic Interpretation (in:) Danny SteInberg, Leon Jacobovits, Semantics, an Interdisciplinary Reader in Philosophy, Linguistics and Psychology, Cambridge, Cambridge University Press, pp. 183-216.

CREIDER, Chet - HudSON, Richard (1999), Inflectional morphology in Word Grammar, Lingua 107 (3-4), Amsterdam, North-Holland Publishing Company, pp. 163-187.

Dalrymple, Mary - Hayrapetian, Irene - Holloway-King, Tracy (1998), The Semantics of the Russian Comitative Construction, Natural Language and Linguistic Theory 16 (3), Dodrecht, Kluwer Academic Publishers, pp. 597-631. 
FLeISCHMAN, Suzanne (1982), The Future in Thought and Language: diachronic evidence from Romance, Cambridge, Cambridge University Press.

GIRY-SCHNEIDER, Jacqueline (1997), Sur quoi peut porter un adjectif épithète ? L'expression du temps et de l'aspect dans les groupes nominaux, Langages 126, Paris, Larousse, pp. 11-38.

JosePh, Brian D. (1998), Diachronic Morphology (in:) Andrew SPENCER, Arnold M. ZWICKY, The Handbook of Morphology, Oxford-Malden, Blackwell Publishers, pp. 351-373.

KarolaK, Stanisław (1965), Przypadek a przyimek, Biuletyn Polskiego Towarzystwa Językoznawczego XXIII, Wrocław, Zakład Narodowy im. Ossolińskich, pp. 143-158.

KAROLAK, Stanisław (2002), Podstawowe struktury składniowe języka polskiego, Warszawa, Slawistyczny Ośrodek Wydawniczy (SOW) przy Instytucie Slawistyki PAN.

KeEnAN, Edward L. (1985), Passive in the World's Languages (in:) Timothy SHOPEN, Language Typology and Syntactic Description. Clause Structure (vol. 1), Cambridge, Cambridge University Press, pp. 243-281.

KeENAN, Edward L. (2002), Some Properties of Natural Language Quantifiers: Generalized Quantifier Theory, Linguistics and Philosophy 25 (5-6), Dodrecht, Kluwer Academic Publishers, pp. 627-654.

KILBURY, James (2001), German noun inflection revisited, Journal of Linguistics 37, Cambridge, Cambridge University Press, pp. 339-353.

KowALIK, Krystyna (1997), Struktura morfonologiczna współczesnej polszczyzny, Kraków, Polska Akademia Nauk-Instytut Języka Polskiego.

KRĄPIEC, Mieczysław Albert (1985), Język $i$ świat realny, Lubin, Wydawnictwo Katolickiego Uniwersytetu Lubelskiego.

LAMIROY, Béatrice (1999), Auxiliaires, langues romanes et grammaticalisation, Langages 135, Paris, Larousse, pp. 33-45.

LEFEUVRE, Florence (1999), La phrase averbale en français, Paris-Montréal, L'Harmattan.

LEISS, Elisabeth (1997), Synkretismus und Natürlichkeit, Folia Linguistica. Acta Societatis Linguisticae Europaeae XXXI (1-2), Berlin, Mouton de Gruyter, pp. 133 -160.

NKOLLO, Mikołaj (2003), Les exposants morphologiques et syntaxiques de la symétrie en français, Studia Romanica Posnaniensa XXX, Poznań, Wydawnictwo UAM, pp. 107122.

SEILER, Hansjakob (1967), On Paradigmatic and Syntagmatic Similarity, Lingua 18, Amsterdam, North-Holland Publishing Company, pp. 35-79.

SERBAT, Guy (1989), Le syncrétisme des cas: Quelques réflexions (in:) Gualtiero CALBOLI, Subordination and other topics in Latin. Proceedings of the Third Colloquium on Latin Linguistics, Bologna, 1-5 April 1985, Amsterdam, John Benjamins Publishing Company (Studies in Language Companion Series 17), pp. 273286.

STUMP, Gregory T. (1998), Inflection (in:) Andrew SPENCER, Arnold M. ZWICKY, The Handbook of Morphology, Oxford-Malden, Blackwell Publishers, pp. 13-43.

STUMP, Gregory T. (2001), Inflectional Morphology: A Theory of Paradigm Structure, Cambridge, Cambridge University Press (Cambridge Studies in Linguistics 93).

WINTER, Yoad (2002), Atoms and Sets: A Characterization of Semantic Number, Linguistic Inquiry 33 (3), Cambridge, Massachusetts, The MIT Press, pp. 493-505.

ŻUKROWSKA, Aleksandra (1998), Struktura i przedmiot wypowiedzi, Studia Semiotyczne XXI-XXII, Wrocław, Zakład Narodowy im. Ossolińskich, pp. 69-78 


\begin{abstract}
This paper surveys the morphological status of converse relations and of symmetric expressions. Thus far, they have received merely cursory descriptions underlining either solely their formal or solely their semantic properties. It is argued that, despite formal differences, these expressions do not alter the meaning of a sentence they give birth to. However, its functional structure (topic / comment partition) may be altered due to their use. From this point of view, converse relations resemble inflectional modifications (such as voice in verbal morphology, degree within adjectives, etc.) undergone by variable lexical items. Moreover, these expressions imply syntactic alternations (pertaining to word order) within sentence's structure. It might be concluded, then, that converse relations are instances of suppletive inflection within diathetic paradigm of a given language. As far as symmetric expressions are concerned, they may, on the same grounds, be described as instances of diathetic syncretism.
\end{abstract}

